\title{
Characteristics of comorbidities and costs among patients who died from systemic lupus erythematosus in Taiwan
}

\author{
Shih-Chao Kang ${ }^{1,2}$, Shinn-Jang Hwang ${ }^{2,3}$, Yu-Sheng Chang, ${ }^{4}$ Chung-Tei Chou ${ }^{2,4}$, Chang-Youh Tsai ${ }^{2,4}$
}

1Division of Family Medicine, National Yang-Ming University Hospital, Yilan, Taiwan 2Faculty of Medicine, National Yang-Ming University School of Medicine, Taipei, Taiwan

32Department of Family Medicine, Taipei Veterans General Hospital, Taiwan

${ }^{4}$ Division of Allergy, Immunology and Rheumatology, Department of Medicine, Taipei Veterans General Hospital, Taiwan

Submitted: 4 November 2011

Accepted: 11 March 2012

Arch Med Sci 2012; 8, 4: 690-696

DOI: 10.5114 /aoms.2012.30293

Copyright $\odot 2012$ Termedia \& Banach

\section{Abstract}

Introduction: Systemic lupus erythematosus (SLE) is prevalent among young female adults, particularly in Asia. In Taiwan, features of end-of-life SLE patients remain unclear.

Material and methods: Data regarding SLE patients whose hospitalization ended up with death were collected and analyzed from the repository of the National Health Insurance Research Database, Taiwan, from 2005 to 2007.

Results: A total of 302 subjects were enrolled and the majority of these were young to middle-aged women ( $45.8 \pm 18.5$ years); only one third of them were treated by rheumatologists. Eight patients $(2.6 \%)$ with comorbid cancers received hospice care. Sepsis/bacteremia (42.1\%) was the major acute comorbidity. Nephropathy/nephritis (35.1\%) represented the major chronic comorbidity. Among 27 subjects with comorbid cancers, gynecological cancers were the most common (18\%). Among the inpatient costs, the cost of prescriptions accounted for the majority $(21.7 \pm 11.5 \%)$. Under a multivariate logistic regression, advanced age ( $\geq 65$ years) correlated positively with acute lower respiratory conditions (ALRC) and diabetes mellitus (DM), and male gender correlated negatively with nephropathy/nephritis. The nephropathy/nephritis correlated positively with hospital stays $>14$ days. The ALRC was closely associated with acute respiratory failure, but not with shock. However, shock was closely associated with hospital stays $\leq 14$ days and sepsis/bacteremia. Cancer development was inversely correlated to nephropathy/nephritis, acute respiratory failure, and shock (all $p<0.05$ ).

Conclusions: The end-of-life SLE patients revealed aforementioned characteristics and relationships. Sepsis/bacteremia, acute respiratory failure, and ALRC contributed most frequently to the ultimate death of acutely ill SLE patients.

Key words: comorbidity, retrospective study, impending death, systemic lupus erythematosus.

\section{Introduction}

Systemic lupus erythematosus (SLE) is prevalent among young women, particularly in non-Caucasian populations. Patients are frequently hospitalized as they suffer from pneumonias or urinary tract infections

\author{
Corresponding author: \\ Prof. Chang-Youh Tsai \\ Division of Allergy, \\ Immunology and Rheumatology \\ Department of Medicine \\ Prof. Shinn-Jang Hwang \\ Department of Family Medicine \\ Taipei Veterans \\ General Hospital \\ 201 Shih-Pai Road Sec. 2 \\ Taipei 112, Taiwan, ROC \\ Phone: +886-2-28757460 \\ Fax: +886-2-28737901 \\ E-mail: cytsai@vghtpe.gov.tw, \\ sjhwang@vghtpe.gov.tw
}


originating from Gram-negative bacteria [1]. In the USA, the increase in the number and the cost of avoidable hospitalizations has been remarkable among SLE patients [2]. Regarding mortality, a Mexican report has revealed a correlation between active lupus nephritis (LN) and infections [3]. A nationwide claim-based study in the USA has found that African Americans have higher mortality due to SLE than Caucasians [4]. Ward et al. reported the predictors of mortality such as age, sepsis, LN, respiratory failure and other indices among inpatients with SLE [5]. It is reported that lower socio-economic status correlated with in-hospital mortality rate of patients with SLE [6]. For genetic issues, the relationship between some genetic mutations and development of SLE has been discussed [7].

In Taiwan, National Health Insurance (NHI), which was launched in 1995, categorizes SLE as a catastrophic illness. According to the standard diagnostic criteria set forth by the American College of Rheumatology (ACR) [8,9], those who are identified by clinical rheumatologists as having SLE as a catastrophic illness are exempt from the cost incurred by this disease in ambulatory as well as in-hospital cares [10]. Therefore, a complete registry of SLE has been established since then. A Taiwanese epidemiological study has revealed a trend of increasing prevalence but a declining incidence of SLE, and its incidence and prevalence in Taiwan were both higher than those in Western countries [11]. Another claim-based study has reported a significant role of experienced physicians in care of SLE patients, which can reduce inhospital mortality from the disease [12]. However, the end-of-life issues of SLE patients in Taiwan remain unclear.

The aim of this investigation was to delineate aforementioned issues from the repository of the database in $\mathrm{NHI}$.

\section{Material and methods}

\section{Design of the study}

Retrospective and claim-based analyses were conducted by collecting data regarding end-of-life hospitalization of patients with SLE from the National Health Insurance Research Database (NHIRD) from 2005 to 2007. The NHIRD is maintained by the National Health Research Institute, Taiwan, and provides anonymous/encrypted NHI claim data to local academic institutes for related studies [13]. Hospitalization claim data (DD 2005.DAT, DD 2006.DAT, and DD2007.DAT) were screened with the International Classification of Diseases, the $9^{\text {th }}$ Revision Clinical Modification (ICD-9-CM) code of 710.0. The reason for discharge included in-hospital death, suicide, and critical discharge for home death (which is a ritual

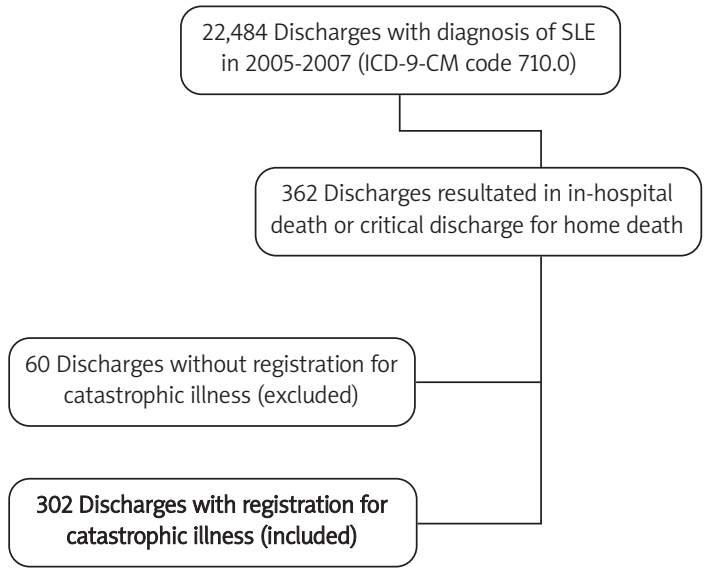

Figure 1. Algorithm for case collection

belief of getting peace in another world abided by many Taiwanese). For accuracy of coding, those who were not registered with catastrophic illness in the $\mathrm{NHI}$ were excluded from the study. The study design is shown in Figure 1.

\section{Parameters}

Subjects' general data, comorbidities and claims for charges were analyzed. The details of inpatients' costs were converted into United States Dollars (USD) according to the closing exchange rate on December 31, 2007. The comorbidities were collected according to related ICD-9-CM codes, which included sepsis or bacteremia (code 038.x, 790.7), acute respiratory failure (code 518.81), non-traumatic shock (code 785.5x), acute myocardial infarction (410.x, 411.x), acute cerebrovascular accident (code 430.x-434.x), acute low respiratory conditions (ALRC) (code 466.x, 480.x-486.x), urological infections (code 590.x, 595.x, 597.x, 599.x, 601.x), peptic ulcer or esophagitis (code 530.x-534.x), hypertensive diseases (code 401.x-405.x), heart failure (code 428.x), diabetes mellitus (DM, code 250.x), cancers (code 140.x208.x) and nephropathy/nephritis (code 580.x-589.x, 791.0). Psychiatric disorders were not included due to possible underestimation of ICD coding in end-oflife hospitalizations. The study was conducted after approval by the National Health Research Institute, Taiwan (No. 99237). All the identity data of subjects was encrypted and analyzed anonymously.

\section{Statistical analysis}

Data in the text and tables are expressed as mean \pm SD. SPSS software (version 17.0, SPSS Inc., Chicago, IL, USA) was used for multivariate logistic regression analysis to assess statistical significance. The correlated factors were presented as regression coefficient (B), odds ratio (OR) and $95 \%$ confidence interval $(\mathrm{Cl})$. A $p$ value of less than 0.05 was considered statistically significant (2-tailed tests). 
Table I. The prevalence and incidence of SLE in 2005 and 2006 in Taiwan

\begin{tabular}{|c|c|c|c|c|c|}
\hline $\begin{array}{l}\text { Date } \\
\text { (year-month-day) }\end{array}$ & Population & $\begin{array}{l}\text { Identified cases } \\
\quad\left(\text { cases } / 10^{5}\right)\end{array}$ & Prevalence & New cases & $\begin{array}{c}\text { Incidence } \\
\text { (cases } / 10^{5} \text { ) }\end{array}$ \\
\hline 2005-1-1 & NA & 14,995 & NA & NA & NA \\
\hline $2005-12-31$ & $22,722,559$ & 15,753 & 69.33 & 758 & 3.34 \\
\hline 2006-1-1 & NA & 15,753 & NA & NA & NA \\
\hline $2006-12-31$ & $22,814,639$ & 16,444 & 72.08 & 691 & 3.03 \\
\hline
\end{tabular}

Table II. Demographic characteristics of patients with SLE who died in or just after the last admission during 2005-2007 ( $N=302)$

\begin{tabular}{|c|c|}
\hline Items & Counts [\%] \\
\hline Mean age $\left[\right.$ years] ${ }^{*}$ & $45.8 \pm 18.5$ \\
\hline Mean hospital stay [days] & $20.4 \pm 18.1$ \\
\hline Gender (male/female) & $41 / 261$ \\
\hline Hospice care (yes/no) & $8 / 294$ \\
\hline \multicolumn{2}{|c|}{ Departments to which admissions were assigned } \\
\hline Family medicine & $4(1.3)$ \\
\hline Internal medicine (general internal) & $33(10.9)$ \\
\hline Internal medicine (rheumatology) & $95(31.5)$ \\
\hline Internal medicine (other specialties) & $119(39.4)$ \\
\hline Surgery & $27(9.0)$ \\
\hline Pediatrics & $14(4.6)$ \\
\hline Neurology & $6(2.0)$ \\
\hline Other sections & $4(1.3)$ \\
\hline
\end{tabular}

\section{Results}

From 2005 to 2007, a total of 22,484 hospital discharges regarding patients with SLE were recorded; three hundred and two mortality discharges fulfilling the criteria for this study were included. The total number of patients holding a catastrophic certificate with a code of 710.0 during 2005 to 2006 was retrieved. No case was registered as suicide as the result of end-of-life hospitalization. As shown in Table I, estimated incidence and prevalence of
SLE during these two years were $3.34 / 10^{5}, 3.03 / 10^{5}$ and $69.33 / 10^{5}, 72.08 / 10^{5}$ respectively.

Table II shows the demographic characteristics of these dead patients with SLE $(N=302)$. The majority of patients were young to middle-aged ( $45.8 \pm 18.5$ years) women (86.4\%), who were admitted to non-hospice (acute) wards (97.4\%) affiliated to Internal Medicine ( $81.8 \%$; including rheumatology, which accounted for $31.5 \%$ of all subjects).

The average inpatient cost for each admission, its detailed items and ratios to the total costs, are listed in Table III. Drug prescriptions represented the majority of the total cost of hospitalization $(21.7 \pm 11.5 \%)$, followed by laboratory examinations (12.4 $\pm 7.6 \%)$.

Table IV lists the major comorbidities. Sepsis/bacteremia (42.1\%) and acute respiratory failure (38.7\%) were the most common acute comorbidities and nephropathy/nephritis (35.1\%) accounted for the majority of chronic comorbidities. Among 27 patients with co-existing cancers, eight received hospice care. Gynecological cancers (5 subjects, $18.5 \%$ of those with cancers; $1.7 \%$ of the total), lung cancers ( 4 subjects, $14.8 \%$ of those with cancers; $1.3 \%$ of the total), colorectal cancers, leukemia/lymphoma and metastatic cancer with unknown origin (3 subjects in each category, $11.1 \%$ of those with cancers; $1.0 \%$ of the total) accounted for approximately half of those with co-existing cancers.

As demonstrated in Table $V$, elderly patients tended to be men $(B=1.022, \mathrm{OR}=2.778)$ and to have $\mathrm{DM}$ $(B=1.935, \mathrm{OR}=6.926)$ and $\operatorname{ALRC}(B=0.880, \mathrm{OR}=$ 2.411) more frequently but to have nephropathy/ nephritis less frequently $(B=-0.974, \mathrm{OR}=0.377$ ) as analyzed by multivariate logistic regression. Nephropa-

Table III. Costs of the end-of-life hospitalization incurred for patients with SLE during 2005-2007 (N=302)

\begin{tabular}{|lcc|}
\hline Items & Costs [USD] & Ratio to total costs [\%] \\
\hline Laboratory tests & $964.7 \pm 935.1$ & $12.4 \pm 7.6$ \\
\hline Radiological examinations & $276.9 \pm 375.4$ & $3.7 \pm 5.8$ \\
\hline Blood products for transfusion & $429.9 \pm 819.5$ & $3.7 \pm 6.3$ \\
\hline Hemodialysis (excluding peritoneal dialysis) & $1,204.5 \pm 2115.7$ & $9.7 \pm 9.6$ \\
\hline Drug prescriptions & $2,224.4 \pm 2929.0$ & 100 \\
\hline Total & $9,530.2 \pm 10354.2$ & 21.5 \\
\hline
\end{tabular}

*USD, United States dollars 
thy/nephritis correlated positively with hospital stays of more than 14 days $(B=0.566, O R=1.761)$. The ALRC was closely associated with acute respiratory failure $(B=0.733, \mathrm{OR}=2.081)$, but not with non-traumatic shock $(B=-0.855$, OR $=0.425)$. Indeed, nontraumatic shock tended to be associated with shorter hospital stays ( $\leq 14$ days, $B=0.901$, $O R=2.463$ ) and sepsis/bacteremia $(B=1.852, \mathrm{OR}=6.369)$. On the other hand, cancers occurred less frequently in subjects with nephropathy/nephritis $(B=-2.006$, $\mathrm{OR}=0.135)$, acute respiratory failure $(B=-1.092$, $\mathrm{OR}=0.336)$, or non-traumatic shock $(B=-2.716$, $\mathrm{OR}=0.113)$.

\section{Discussion}

For demographic characteristics and the role of rheumatologists

As demonstrated in Table II, the majority of patients were young females, consistent with previous reports. Patients were usually admitted to the acute wards and were cared for by internal physicians, indicating that complications of lupus disease often develop acutely and are unlikely to require surgical intervention. However, it should be noted that rheumatologists only covered around one third of dead SLE patients for their end-of-life hospitalizations in Taiwan. This phenomenon indicated that SLE itself is manifested with multi-organ involvement and damage. Thus, patients with SLE were admitted with lethal complications from different involved organs/systems, for which the specialists were assigned to care. Our results suggest that internal physicians should have relevant knowledge and capacity to care for patients with SLE, and rheumatologists should take more responsibility to consult or combine care for complicated SLE patients in Taiwan.
Table IV. Major comorbidities in the end-of-life hospitalization of patients with SLE during 2005-2007 $(N=302)$

\begin{tabular}{|lc|}
\hline Comorbidities & No. (\%) \\
\hline Sepsis or bacteremia & $127(42.1)$ \\
\hline Acute respiratory failure & $117(38.7)$ \\
\hline Shock, non-traumatic & $63(20.9)$ \\
\hline Acute myocardial infarction & $8(2.6)$ \\
\hline Acute cerebrovascular accident & $31(10.3)$ \\
\hline Acute lower respiratory conditions & $81(26.8)$ \\
\hline Urological infections & $23(7.6)$ \\
\hline Peptic ulcer or esophagitis & $12(4.0)$ \\
\hline Hypertensive diseases & $32(10.6)$ \\
\hline Heart failure & $23(7.6)$ \\
\hline Diabetes mellitus & $15(5.0)$ \\
\hline Cancers & $27(8.9)$ \\
\hline Nephropathy/nephritis & $106(35.1)$ \\
\hline
\end{tabular}

\section{For the costs and pathway of care in end-of-life SLE}

Regarding the medical costs for SLE, Pelletier et al. reported an impact of co-existing nephropathy on higher inpatient and outpatient expenses [14]. In the USA, the inpatient costs for SLE have increased to a mean of 10,000 USD for each admission [6]. In Taiwan, the mean inpatient cost for SLE was lower than those in the USA, totaling 1,923 USD for each admission [10]. In this study, the mean cost for each end-of-life hospitalization was more expensive $(9,530 \pm 10,354$ USD) than those report-

Table V. Correlation of individual factors with each other in SLE patients approaching death, calculated by multivariate logistic regression

\begin{tabular}{|llcccc|}
\hline Dependent variable & Covariates & B & OR & $95 \% \mathrm{Cl}$ & Value of $p$ \\
\hline Age $\geq 65$ years & Nephropathy/nephritis & -0.974 & 0.377 & $0.181-0.789$ & 0.010 \\
\cline { 2 - 6 } & ALRC & 0.880 & 2.411 & $1.282-4.535$ & 0.006 \\
\cline { 2 - 6 } & Diabetes mellitus & 1.935 & 6.926 & $2.264-21.187$ & 0.001 \\
\cline { 2 - 6 } & Male gender & 1.022 & 2.778 & $1.300-5.935$ & 0.008 \\
\hline Nephropathy/nephritis & Hospital stay > 14 days & 0.566 & 1.761 & $1.080-2.871$ & 0.023 \\
\cline { 2 - 6 } & Cancers & -2.006 & 0.135 & $0.031-0.582$ & 0.007 \\
\hline Acute respiratory failure & ALRC & 0.733 & 2.081 & $1.235-3.506$ & 0.006 \\
\cline { 2 - 6 } & Cancers & -1.092 & 0.336 & $0.122-0.921$ & 0.034 \\
\hline Shock, non-traumatic & Hospital stay $\leq 14$ days & 0.901 & 2.463 & $1.312-4.608$ & 0.005 \\
\cline { 2 - 6 } & ALRC & -0.855 & 0.425 & $0.194-0.933$ & 0.033 \\
\cline { 2 - 6 } & Sepsis or bacteremia & 1.852 & 6.369 & $3.304-12.277$ & $<0.001$ \\
\cline { 2 - 6 } & Cancers & -2.716 & 0.113 & $0.014-0.911$ & 0.041 \\
\hline
\end{tabular}

$B$ - regression coefficient, $O R$ - odds ratio, $C l$ - confidence interval, $A L R C$ - acute lower respiratory condition 
ed in the aforementioned epidemiological studies. Regarding the details of cost, a large proportion was allocated to drug prescriptions to maintain vital organs as well as the laboratory tests accompanying these tasks. It is conceivable that late complications of SLE are so extensive and catastrophic that they would account for most of the life-long treatment costs. Interestingly, subjects who received hospice care had significantly lower total costs for end-of-life hospitalization than the others (1,664 $\pm 3,014$ vs. 9,744 $\pm 10,401$ USD, $p<0.001$ by two-sample $t$-test). The cost-saving benefits of hospice care have been confirmed by previous studies and might be considered as an alternative way to care for terminal SLE patients [15-17].

\section{For acute/chronic comorbidities in end-of-life} SLE

The acute comorbidities in end-of-life SLE patients provided clues to the mortality issues. In other countries or areas, previous epidemiological and hospital-based studies have identified infections as the leading cause of death in SLE patients [18-20]. In Taiwan, Feng et al. reported that inadequate use of antibiotics and prolonged hospital stays increased the mortality of SLE patients in intensive care units [21]. Similarly, our results demonstrated that sepsis/bacteremia, acute respiratory failure and ALRC were the three most common acute comorbidities in dying SLE patients. These conditions are likely to have a major impact on SLE patients during their end of life. We supposed that hematogenous infections were the most difficult factor to deal with in terms of treating patients with life-threatening SLE.

Among the chronic comorbidities, nephropathy/nephritis prevailed in dying SLE patients. This was consistent with reports showing a high prevalence of lupus renal diseases in Orientals [22]. Another study has also reported an increasing trend of Medicare expense in parallel to the increasing incidence of renal damage in SLE patients [23]. In this study, we can see that nephropathy/nephritis in SLE patients claimed significantly higher total expenses for hospitalization, particularly during the end of life $(11,710 \pm 10,885$ vs. $8,351 \pm 9,885, p=0.007$ by two-sample $t$-test), although relatively longer hospital stay might be a confounding factor. This suggests that early prevention or treatment of nephropathy/nephritis is an essential requirement to save costs that may be ultimately incurred by prolonged and expensive treatment for catastrophic complications of SLE.

Our results have demonstrated that $5 \%$ of dying SLE patients had concurrent DM, a figure similar to those reported elsewhere. It has been widely accepted that patients with SLE have a higher risk for metabolic syndrome, coronary artery disease and/or stroke [24, 25]. Our results showed a higher prevalence of DM in male SLE patients approaching death $(7.3 \%$ vs. $4.7-6.5 \%$ in general male population; $4.6 \%$ vs. $5.3-6.6 \%$ in general female population) in Taiwan [26]. Also, the prevalence of acute cerebrovascular accidents in our patients was higher than those reported previously $[27,28]$. On the other hand, occurrence of acute myocardial infarction was similar to those reported endemically [29]. These have suggested that prolonged inflammation or autoimmune processes can lead to the development of vascular damage that subsequently results in fatal cerebrovascular or cardiovascular episodes.

\section{For relationships of logistic regressions}

Significant associations or correlations have been observed in this study. For example, older patients tended to have more chance of getting diabetes, patients with ALRC were more prone to acute respiratory failure, and patients with non-traumatic shock tended to have sepsis/bacteremia as their cause. We supposed that these conditions could be induced by glucose intolerance and immunosuppressive status after long-term use of corticosteroids. In addition, it is interesting that nephropathy was more frequently identified in younger patients and associated with a prolonged hospital stay. Regarding the latter, no compelling data could support this finding up to now. It needs further clarification. Regarding the former, Pelletier et al. demonstrated a similar risk in American SLE populations [14]. Another Canadian study also reported a higher incidence of nephropathy in younger patients with early onset of illness in non-Caucasian pediatric SLE patients [30]. Furthermore, more rapid progression of nephropathy and lower survival rates were reported in non-Caucasian SLE patients [31]. Our results demonstrated a similar characteristic of nephropathy/nephritis, including LN. This suggests that earlier diagnosis and control of nephropathy are essential for reducing mortality rates and medical costs in SLE patients.

Non-traumatic shock was associated with shorter hospital stays and fewer ALRC. The most likely cause of shorter hospitalization time was rapid deterioration course resulting in death in these immunosuppressive patients. This reflected the impact of shock on the progression of the illness. The association between fewer ALRC and more nontraumatic shock implied that the major pattern of infections might be sepsis/bacteremia without specific origins in critical SLE patients due to their poorer immunosuppressive status. Actually, in many patients dying of septic shock the original source of infection could not be definitely identified. So, occult infections should be looked for and carefully evaluated for every critical SLE patient [32]. 
Another interesting issue was that elderly patients (age $\geq 65$ years) tended to be male and to have ALRC. A previous local epidemiological study reported a definitely better prognosis in young-middle aged female SLE patients and a lower survival rate of male SLE patients in Taiwan [10]. The reasons for the discrepancy with previous reports are unknown. It is possible that male patients with SLE have been overlooked and underestimated in Taiwan owing to a low index of suspicion by clinical practitioners other than rheumatologists because of overwhelming comorbidities, and by patients and their families. The higher ratio of ALRC implied that respiratory tract infections constitute a major problem in elderly SLE patients. Relevant studies were rare in aged SLE patients because their counts were limited. Further extensive cohort investigations may be necessary to clarify this point.

The understanding of the implications and pathogenic relevance of comorbid cancers in SLE has changed in the past decade. Recent studies have demonstrated an increased incidence of cervical, bladder, lung and hematological cancers in SLE patients [33, 34]. Chen et al. have reported an overall increased risk of cancers in Taiwanese SLE patients [35]. Our results demonstrated similar features in critical SLE patients, although the number of patients enrolled was much lower than in other investigations. The reason why these comorbid cancers involve the hematological, respiratory and genitourinary systems is unknown. Further in-depth investigation is mandatory to determine whether long-term immunosuppressive medications or similar immunopathogenetic mechanisms with SLE caused comorbid cancers. On the other hand, the absence of a correlation between cancer incidence and some acute illnesses in dying SLE patients suggests that lethal infections may be more important factors in causing death than associated cancers. Similarly, the lack of association between cancers and chronic illness such as nephropathy/nephritis has indicated that renal inflammation may not be an essential factor for the development of malignancies in aged SLE patients. Further investigations may be necessary to clarify the issue.

There were a few limitations in this study. Possible errors in registration of the patients are unavoidable in a large population of patients like this. Absence of detailed relevant laboratory data, specific serum markers and records of immunosuppressive prescriptions in claim data restricted the power of the study to evaluate the activity or severity of SLE itself. Absence of linkage with a mortality dataset also limited the in-depth approach to cause of death due to official and legal limitations. LN could not be discussed separately from nephropathy/nephritis owing to limitations of present ICD-9-CM coding. Nevertheless, the present investigation constitutes a novel approach to study the SLE patients on the verge of death on a nationwide scale. Further case-controlled or cohort studies are necessary for a more in-depth understanding of the natural course of SLE.

In conclusion, a survey of the end-of-life hospitalization of patients with SLE has identified the various characteristics of these patients during the end stage of the disease. Sepsis/bacteremia, acute respiratory failure, and ALRC were common acute co-morbidities in dying SLE patients. Nephropathy/nephritis mainly manifested in younger subject. Elderly SLE patients presented with unique characteristics during the last period of survival, which require further assessment. The difficulties in the care of SLE inpatients challenge healthcare providers, who require more awareness to reduce disease per se or complications in dying SLE patients.

\section{Acknowledgments}

The authors wish to thank the National Health Research Institute for permission and authorization to utilize the dataset. The first author wrote this article in memory of his late mother, Madam Jing-Mei Chuang, who passed away with end-stage LN and iatrogenic infections. This study does not represent the official viewpoints of the National Health Research Institute, Taiwan. The work was supported in part by grants from the National Science Council Taiwan (NSC97-2314-B-075-009-MY3) and Taipei Veterans General Hospital (V99C1-129). The authors declare no conflict of interest about this study.

\section{References}

1. Navarro-Zarza JE, Alvarez-Hernández E, Casasola-Vargas JC, et al. Prevalence of community-acquired and nosocomial infections in hospitalized patients with systemic lupus erythematosus. Lupus 2010; 19: 43-8.

2. Ward MM. Avoidable hospitalizations in patients with systemic lupus erythematosus. Arthritis Rheum 2008; 59: 162-8

3. Zonana-Nacach A, Yañez P, Jiménez-Balderas FJ, et al. Disease activity, damage and survival in Mexican patients with acute severe systemic lupus erythematosus. Lupus 2007; 16: 997-1000.

4. Krishnan E, Hubert HB. Ethnicity and mortality from systemic lupus erythematosus in the US. Ann Rheum Dis 2006; 65: 1500-5.

5. Ward MM, Pajevic S, Dreyfuss J, et al. Short-term prediction of mortality in patients with systemic lupus erythematosus: classification of outcomes using random forests. Arthritis Rheum 2006; 55: 74-80.

6. Krishnan E. Hospitalization and mortality of patients with systemic lupus erythematosus. J Rheumatol 2006; 33: 1770-4.

7. Skrętkowicz J, Barańska M, Kaczorowska A, RychlikSych M. Genetic polymorphisms of CYP2D6 oxidation in patients with systemic lupus erythematosus. Arch Med Sci 2011; 7: 864-9. 
8. Tan EM, Cohen AS, Fries JF, et al. The 1982 revised criteria for the classification of systemic lupus erythematosus. Arthritis Rheum 1982; 25: 1271-7.

9. Hochberg MC. Updating the American College of Rheumatology revised criteria for the classification of systemic lupus erythematosus. Arthritis Rheum 1997; 40: 1725.

10. Special Medical Needs, Bureau of National Health Insurance, Department of Health, Executive Yuan, Republic of China (Taiwan), 2012. Available at: http://www.nhi.gov.tw/english/index.aspx.

11. Chiu YM, Lai CH. Nationwide population-based epidemiologic study of systemic lupus erythematosus in Taiwan. Lupus 2010; 19: 1250-5.

12. Wang TF, Lin HC. Relationship between caseload volume and outcome for systemic lupus erythematosus treatment: the experience of Taiwan. J Rheumatol 2008; 35: $1795-800$.

13. National Health Insurance Research Database, National Health Research Institutes, Republic of China (Taiwan), 2012. Available at: http://w3.nhri.org.tw/nhird/en/index.htm.

14. Pelletier EM, Ogale S, Yu E, et al. Economic outcomes in patients diagnosed with systemic lupus erythematosus with versus without nephritis: results from an analysis of data from a US claims database. Clin Ther 2009; 31: 2653-64.

15. Lin WY, Chiu TY, Hsu HS, et al. Medical expenditure and family satisfaction between hospice and general care in terminal cancer patients in Taiwan. J Formos Med Assoc 2009; 108: 794-802.

16. Paz-Ruiz S, Gomez-Batiste X, Espinosa J, et al. The costs and savings of a regional public palliative care program: the Catalan experience at 18 years. J Pain Symptom Manage 2009; 38: 87-96.

17. Bendaly EA, Groves J, Juliar B, et al. Financial impact of palliative care consultation in a public hospital. J Palliat Med 2008; 11: 1304-8.

18. Abu-Shakra M, Urowitz MB, Gladman DD, et al. Mortality studies in systemic lupus erythematosus. Results from a single center. I. Causes of death. J Rheumatol 1995; 22: 1259-64.

19. Gulay CB, Dans LF. Clinical presentations and outcomes of Filipino juvenile systemic lupus erythematosus. Pediatr Rheumatol Online J 2011; 9: 7.

20. Mok C. Epidemiology and survival of systemic lupus erythematosus in Hong Kong Chinese. Lupus 2011; 27: 767-71.

21. Feng $\mathrm{PH}$, Lin $\mathrm{SM}, \mathrm{Yu} \mathrm{CT}$, et al. Inadequate antimicrobial treatment for nosocomial infection is a mortality risk factor for systemic lupus erythematous patients admitted to intensive care unit. Am J Med Sci 2010; 340: 64-8.

22. Crosslin KL, Wiginton KL. The impact of race and ethnicity on disease severity in systemic lupus erythematosus. Ethn Dis 2009; 19: 301-7.

23. Clarke AE, Panopalis $P$, Petri $M$, et al. SLE patients with renal damage incur higher health care costs. Rheumatology (Oxford) 2008; 47: 329-33.

24. Mok CC, Poon WL, Lai JP, et al. Metabolic syndrome, endothelial injury, and subclinical atherosclerosis in patients with systemic lupus erythematosus. Scand J Rheumatol 2010; 39: 42-9.

25. Hak AE, Karlson EW, Feskanich D, et al. Systemic lupus erythematosus and the risk of cardiovascular disease: results from the nurses' health study. Arthritis Rheum 2009; 61: 1396-402.

26. Chang $\mathrm{CH}$, Shau WY, Jiang YD, et al. Type 2 diabetes prevalence and incidence among adults in Taiwan during
1999-2004: a national health insurance data set study. Diabet Med 2010; 27: 636-43.

27. Lin HC, Lin YJ, Liu TC, et al. Urbanization and stroke prevalence in Taiwan: analysis of a nationwide survey. J Urban Health 2007; 84: 604-14.

28. Huang ZS, Chiang TL, Lee TK. Stroke prevalence in Taiwan. Findings from the 1994 National Health Interview Survey. Stroke 1997; 28: 1579-84.

29. Wang KT, Chen CY, Hou CJ, et al. Comparison of clinical characteristics of acute myocardial infarction in aborigines and nonaborigines in Taitung area of Taiwan. Angiology 2007; 58: 61-6.

30. Hiraki LT, Benseler SM, Tyrrell PN, et al. Ethnic differences in pediatric systemic lupus erythematosus. J Rheumatol 2009; 36: 2539-46.

31. Korbet SM, Schwartz MM, Evans J, et al. Severe lupus nephritis: racial differences in presentation and outcome. J Am Soc Nephrol 2007; 18: 244-54.

32. Zhou WJ, Yang CD. The causes and clinical significance of fever in systemic lupus erythematosus: a retrospective study of 487 hospitalised patients. Lupus 2009; 18 807-12.

33. Bernatsky S, Boivin JF, Joseph L, et al. An international cohort study of cancer in systemic lupus erythematosus. Arthritis Rheum 2005; 52: 1481-90.

34. Kang KY, Kim HO, Yoon HS, et al. Incidence of cancer among female patients with systemic lupus erythematosus in Korea. Clin Rheumatol 2010; 29: 381-8.

35. Chen YJ, Chang YT, Wang CB, et al. Malignancy in systemic lupus erythematosus: a nationwide cohort study in Taiwan. Am J Med 2010; 123: 1150.e1-6. 